\title{
Monkeypox: A Neglected Viral Zoonotic Disease
}

\author{
Moyinoluwa Joshua Oladoye ${ }^{1,2^{*}}$ (D) \\ ${ }^{1}$ Faculty of Veterinary Medicine, University of Ibadan, Ibadan, Oyo State, Nigeria \\ ${ }^{2}$ Slum and Rural Health Initiative Research Academy, Nigeria \\ * Corresponding author: Moyinoluwa Joshua Oladoye E-mail: oladoyemoyinoluwalogo@gmail.com ORCID: 0000-0003-0300-1474 \\ Received: 1 January 2021 Accepted: 16 February 2021
}

\begin{abstract}
Over the years, the nations of the world have been plagued with several infectious diseases outbreak. With the continuous rise in the number of emerging and re-emerging infectious diseases such as monkeypox, it is high time we drew lessons and insights from past outbreaks to guide and effectively prepare for possible future outbreaks. Monkeypox, popularly known as the most important orthopoxvirus infection, is a viral zoonotic disease prevalent in Central and West Africa. There are 2 known genetic clades of the monkeypox virus reported so far, the Western African and the Central African clades. The human monkeypox though originated from Africa has grown to be a global concern with cases reported in the United States, United Kingdom, Israel, and Singapore. It is distinctively characterized by lymphadenopathy and systemic rash symptoms. Monkeypox has a low fatality rate (0-11\%) with the highest rates occurring in children and young persons who were not part of the smallpox vaccinated population. Report suggests that consumption of bush meats could be a potential risk factor in the transmission of the infection or caring for an infected patient. Several factors have been responsible for the weak surveillance system of monkeypox including the current COVID-19 pandemic that has put more pressure on the fragile healthcare systems and infrastructures of most countries. This paper aims to guide global health policymakers on the impending danger of neglecting this disease. The discourse is concluded with the recommendation of strategies to effectively strengthen the disease surveillance system in combating this public health threat.
\end{abstract}

Keywords: monkeypox, smallpox, orthopox, epidemiology, COVID-19

\section{INTRODUCTION}

Monkeypox is a viral disease of public health importance caused by monkeypox virus, a member of orthopoxvirus genus of the family of Poxviridae. It was first isolated in Denmark from vesico-pustular lesions of infected cynomolgus monkeys in 1958 [1]. The monkeys were imported from Singapore. According to the World Health Organization, it is the most important orthopoxvirus infection in humans. It is a viral zoonotic disease that is most prevalent in Central and West Africa. The orthopoxvirus genus also comprises three other species that are pathogenic to humans; they are variola virus, cowpox virus and vaccinia virus [2]. Monkeypox is a closely related disease to smallpox but with a striking distinguishing feature of an early lymphadenopathy. In the western hemisphere, human monkeypox was first isolated in 2003 and has recently experienced an upsurge in the African clime where it is predominantly found since 1970 [3]. This can be associated 
with the increasing interaction between humans and carrier animals of the monkeypox virus. Other factors have proven to be responsible for this such as ecosystem degradation and increased human-to-human rate of transmission [3].

The response to the COVID-19 pandemic has taught countries invaluable lessons about the possible effects of future disease outbreaks that could cause such havoc on lives and livelihoods. In the light of the current pandemic that has put more pressure on the fragile healthcare systems of most countries, there is a need to look at strategies that can help to strengthen the monkeypox disease surveillance system. It is therefore imperative to put structures in place in preparedness for a possible future outbreak of monkeypox. This paper discusses the available data on monkeypox, addresses the challenges of the disease surveillance system hindering the eradication of this public health threat and finally proffers recommendations to policymakers.

\section{REVIEW}

\section{Epidemiology}

Monkeypox has experienced an increased incidence in the last two decades since its first human case discovery in 1970 with high prevalence reported in Nigeria, DRC, Liberia, Cameroon, Gabon, Sudan, Sierra Leone, Republic of the Congo and the Central African Republic [4-9]. In recent years, the sudden incidence in countries like the United States, United Kingdom, Singapore, and Israel have been reportedly linked with the African origin of the disease [10-13].

There are 2 known genetic clades of monkeypox virus reported so far, the Western African and the Central African clades. The Central African clade has a higher case fatality rate $(11 \%)$ than the Western African clade $(<1 \%)$ with an additional human-to-human transmission seen in the Central African clade [14]. The West African clade isolates have been reported in Nigeria, Liberia, Sierra Leone, Ivory Coast and USA while the Central African clade isolates originate from the Republic of Congo, Cameroon, Gabon, Sudan and the DRC [14-17].

According to the World Health Organization, it is commonly found in young persons under the age of 40 or 50 years (varies with country) due to cessation of smallpox vaccination following the eradication of the disease in 1980. Report reveals that most monkeypox cases occur in population under the age of 40 with a median age of 31 years [18]. Asides from the age distribution, the gender distribution of the disease has also been studied. In Nigeria as a case study, monkeypox cases have been recorded in 26 states out of the 36 states of the country (the Federal Capital
Table 1. Comparison of sex distribution and clinical features observed in persons with monkeypox between two countries ( $\mathrm{n}$ for Nigeria=122; $\mathrm{n}$ for United States=30) $[20,22]$

\begin{tabular}{|c|c|c|c|}
\hline \multicolumn{2}{|c|}{ CHARACTERISTIC } & $\begin{array}{c}\text { NIGERIA } \\
\text { (\%) }\end{array}$ & $\begin{array}{c}\text { UNITED } \\
\text { STATES (\%) }\end{array}$ \\
\hline SEX & Female & 31 & 40 \\
\hline $\begin{array}{c}\text { CLINICAL } \\
\text { FEATURES }\end{array}$ & Male & 69 & 60 \\
\hline & Rash & 100 & 83 \\
\hline & Fever & 88 & 73 \\
\hline & Sore throats & 58 & 47 \\
\hline & Headache & 79 & 33 \\
\hline
\end{tabular}

Territory inclusive), an increase to the 11 states initially reported in 2017 [19-21]. This report confirms that the male to female ratio is $3: 1$, suggesting a higher prevalence in the male gender [19]. The male gender is more affected than the female as shown in Table 1.

The largest reservoirs of the virus are rodents, squirrels and giant pouched rats which serve as a source of nutrition in some parts of the world [18]. The disease is primarily transmitted by direct contact with infected reservoir animals. Monkeypox has a low fatality rate (0-11\%) which makes it less severe than smallpox (30-50\%). The highest rates occur in children and young persons who were not part of the smallpox vaccinated population.

In 2003, monkeypox cases were reported from six states during the United States outbreak [22]. The infection was found to have been transmitted by pet prairie dogs suggested to have contracted the virus from small mammals shipped into the US from Ghana [23]. These infected mammals were kept near prairie dogs that were later sold as pets.

In September 2018, human monkeypox experienced another international spread when two travelling Nigerians were diagnosed of the infection in the United Kingdom [10]. A third case was later reported in a healthcare worker who contracted the disease from one of the first two. This is the major evidence available for the human-to-human transmission present in monkeypox.

In October 2018, Israel reported an imported case of monkeypox from her resident who arrived from Nigeria [12]. A similar case was recorded in 2019 when a Nigerian travelled to Singapore. This explains the role travelers play in the spread of infectious diseases epidemics in new regions globally. 


\section{Aetiology}

Monkeypox is caused by Monkeypox virus (MPXV), a member of the orthopoxvirus group of viruses. It is a doublestranded DNA virus. Research suggests the evolutionary nature of the monkeypox virus [24].

The incidence of human infection of monkeypox is hinged on certain risk factors. The most important factor is the discontinuation of smallpox vaccination campaign which has resulted in waning cross-protective immunity [18]. This has made the younger age group the most susceptible to the infection. Another central factor attributed to the increasing incidence is the increased close interaction between humans and the reservoir hosts of the virus [18]. This is a challenge because monkeypox virus can infect a wide range of small mammals (reservoir hosts) such as Sciurid, Glirid and Nesomyid rodents (Cynomys sp., Funisciurussp., Graphiurus sp., Cricetomys sp.), marsupials (Monodelphisdomestica, Delphiusmarsupialis), and primates (Callithrixjacchus, Homo sapiens) [25]. It has also been suggested that consumption of bush meats could be a potential risk factor. Other factors include male gender, civil wars, refugee displacement, farming, deforestation, climate change, demographic changes and population movement $[18,19]$.

Another study suggested common factors responsible for the re-emergence of human monkeypox as climate change, rain forest exploitation, waning herd immunity, transboundary migration and geopolitical conflicts in disease regions [26]. Other indirect causes of rise in prevalence and incidence of monkeypox especially in northern Nigeria includes poverty, ignorance, harmful traditional practices etc. [27-29].

\section{Symptoms}

The incubation period of monkeypox virus varies from 5 to 21 days. The following are common symptoms experienced in individuals infected with monkeypox [30-32]: (1) Fever (2) Rash (3) Sore throat (4) Lymphadenopathy (5) Secondary bacterial infections, (6) Respiratory distress, (7) Bronchopneumonia, (8) Gastrointestinal involvement, (9) Dehydration, (10) Sepsis, (11) Encephalitis, and (12) Corneal infection with ensuing loss of vision.

Data taken from confirmed monkeypox cases in Nigeria and the United States suggests similarities in the prominent clinical features in each region (Table 1). The most prominent feature commonly observed is rash $(100 \%$ and $83 \%$ ) while the least prominent is sore throat (58\% and $33 \%$ ).
Percentages were calculated individually for each characteristic based on the number of patients with available data.

\section{Diagnosis}

Samples are best taken from the skin lesions such as pustules, vesicles and dry crusts after which they are stored in a dry, sterile tube and kept cold. Skin biopsy is a good alternative for sample collection. Arriving at a definitive diagnosis proves quite challenging in the presence of differentials with common symptom of systemic rash illness as seen in measles, bacterial skin infections, scabies, syphilis and medication-associated allergies [24]. The common differentials of monkeypox are orthopoxviruses which include smallpox and chicken pox. A recent study reveals the detection of monkeypox virus in pustular swab through transmission electron microscopy and Polymerase Chain Reaction (PCR) and confirmatory diagnosis by EnzymeLinked Immunosorbent Assay (ELISA), tissue culture and immunofluorescence assay [25].

\section{Virus Characterization}

Monkeypox virus differs from other orthopoxvirus species by the presence of a characteristic pock formation when the virus is inoculated in embryonated hen eggs. Evidence shows that further genomic analyzes aid characterization of the virus isolates and help in categorizing different strains of the monkeypox [24].

\section{Population Sero-surveys and Associated Methods}

Over the years, serological testing has proven effective in identification of monkeypox infection [24]. Moreover, there has been a recent introduction of an anti-orthopoxvirus IgM capture assay invented by the $C D C$ to aid disease incidence capturing [33]. The use of monkeypox-specific serologic assay, the Radioimmunoassay Absorption (RIAA) test is also highly recommended.

\section{Management}

Over the years, there has been no proven treatment available for the treatment of monkeypox infection. However, smallpox vaccine, cidofovir, ST-246, and vaccinia immune globulin (VIG) have been recommended in the management of monkeypox outbreaks. The smallpox vaccination is best administered within two weeks of exposure to monkeypox. Scientific data reveals that smallpox vaccination confers $85 \%$ protection from the disease [34]. The major challenge with the smallpox vaccination is the contraindication in population with high prevalence of HIV infection due to complication risks. 
With no approved anti-viral drug, human monkeypox cases are best managed through symptomatic and supportive therapy [9]. Different anti-viral agents are being developed which may prove useful in mitigating the disease spread. Though not yet available to the public, a new vaccinia-based vaccine was produced in 2019 for the prevention of monkeypox. There is an ongoing study to validate the effectiveness and safety of the Modified Vaccinia Ankara (MVA) vaccine in human cases as it is believed to confer immunity against all orthopoxviruses [35-37].

\section{Challenges of Monkeypox Disease Surveillance System}

It is a known fact that disease surveillance and reporting system is crucial in addressing infectious diseases outbreaks globally. Over the years, there have been several challenges associated with global surveillance data for monkeypox. The common challenges faced include:

- Underreporting due to inadequate access to healthcare facilities in developing countries

- Asymptomatic cases of monkeypox that go undiagnosed

- Poor infectious diseases laboratory support in endemic countries

- Most developing countries where the disease is endemic have a good number of unskilled staff and poor welfare of their healthcare workers

- Countries' unwillingness to give full reports due to the economic and political consequences of such. These consequences range from loss of tourism to possible travel restrictions on affected countries.

- Lack of global coordination, collaboration and solidarity towards achieving global health equity.

Moreover, it is no longer news that the ongoing COVID-19 outbreak has put much pressure on the existing fragile healthcare surveillance systems and infrastructures of countries where monkeypox is endemic. This disruption of access to healthcare might be an indicator that another infectious disease outbreak is on the loose. A recent report suggested a high risk of further spread of the disease in the DRC due to the current COVID-19 pandemic [38]. This undermines the need to critically examine the limitations currently faced in addressing monkeypox infection, especially in the low-and-middle-income countries (LMICs).

\section{Recommendations}

Currently, there are few laboratories equipped with facilities to confirm monkeypox infection in its prevalent regions. This issue of poor diagnostic facilities needs to be addressed to enhance early detection against outbreaks. There is also uncertainty on the natural history of the monkeypox virus. This demands extensive studies to discover the reservoirs of the virus and its transmission cycle. Evidence suggests that implementation of wildlife trade ban could help to curb the migration across regions. Other strategies that help elimination of other prior infectious diseases can be adopted. Furthermore, more epidemiological studies should be conducted to study the prevalence, incidence, morbidity and mortality of monkeypox virus across years in Africa [39]. Low-and-middle-income countries should also pioneer and invest in research to detect possible new viral strains of the disease.

There is a knowledge deficit in the transmission pattern of the disease and associated risks. Adequate funding should be made available for monkeypox research to better understand the zoonotic hosts, reservoirs and vectors of the monkeypox virus [40]. An empirical review on the natural history of the disease will prepare us to respond effectively in cases of future outbreaks. To effectively combat this neglected disease, there is a need to adopt the 'One Health' approach by involving every relevant sector [41].

\section{CONCLUSION}

Monkeypox is a viral zoonotic disease that is predominantly found in Central and West Africa. It has a clinical presentation similar to smallpox which was declared eradicated in 1980. Research reveals the evolutionary nature of the monkeypox virus, the known causative agent of monkeypox. The experience from the ongoing COVID-19 pandemic has shown the world the need to be well prepared against possible future outbreaks/pandemics. Hence, this is the right time to start building structures to strengthen disease surveillance and reporting system as it is crucial in addressing infectious disease outbreaks globally.

Funding: The author received no financial support for the research. Declaration of interest: There is no conflict of interest.

Data availability: Data generated or analysed during this study are available from the author on request.

Acknowledgements: The author acknowledges his colleagues from Slum and Rural Health Initiative Research Academy who provided insight and expertise that greatly assisted the research. 


\section{REFERENCES}

1. Magnus PV, Andersen EK, Petersen KB, Birch-Andersen A. A pox-like disease in cynomolgus monkeys. Acta Pathol Microbiol Scand. 1959;46(2):156-76. (doi: 10.1111/j.16990463.1959.tb00328.x)

2. Shchelkunov SN, Marennikova SS, Moyer RW. Orthopovxiruses Pathogenic for Humans. Chapter: Classification of Poxviruses and Brief Characterization of the Genus. New York, NY: Springer. 2005.

3. Di Giulio DB, Eckburg PB. Human monkeypox: an emerging zoonosis. Lancet Infect Dis. 2004;4(1):15-25. (doi: 10.1016/S1473-3099(03)00856-9).

4. Hutin YJ, Williams RJ, Malfait $P$, et al. Outbreak of human monkeypox, Democratic Republic of Congo, 1996 to 1997. Emerg Infect Dis. 2001;7(3):434. (doi: 10.3201/eid0703.010311).

5. Kalthan E, Tenguere J, Ndjapou SG, et al. Investigation of an outbreak of monkeypox in an area occupied by armed groups, Central African Republic. Med Mal Infect. 2018;48(4):263-8. (doi: 10.1016/j.medmal.2018.02.010).

6. Yinka-Ogunleye A, Aruna O, Ogoina D, et al. Reemergence of human monkeypox in Nigeria, 2017. Emerg Infect Dis. 2018;24(6):1149. (doi: 10.3201/eid2406.180017).

7. Rimoin AW, Mulembakani PM, Johnston SC, et al. Major increase in human monkeypox incidence 30 years after smallpox vaccination campaigns cease in the Democratic Republic of Congo. Proceedings of the National Academy of Sciences. 2010;107(37):16262-7. (doi: 10.1073/pnas.1005769107).

8. Hoff NA, Doshi RH, Colwell B, et al. Evolution of a disease surveillance system: an increase in reporting of human monkeypox disease in the Democratic Republic of the Congo, 2001-2013. Int J Trop Dis Health. 2017;25(2). (doi: 10.9734/IJTDH/2017/35885).

9. Durski KN, McCollum AM, Nakazawa Y, et al. Emergence of monkeypox-west and central Africa, 1970-2017. Morbidity and mortality weekly report. 2018;67(10):306. (doi: 10.15585/mmwr.mm6710a5).

10. Vaughan A, Aarons E, Astbury J, et al. Two cases of monkeypox imported to the United Kingdom, September 2018. Euro Surveill. 2018;23(38):1800509. (doi: 10.2807/1560-7917.ES.2018.23.38.1800509).
11. World Health Organization (WHO). Monkeypox Singapore. WHO Disease outbreak news, May. 2019. [cited 2020 Oct 28]. Available at: https://www.who.int/ csr/don/16-may-2019-monkeypox-singapore/en/

12. Erez $N$, Achdout $H$, Milrot $E$, et al. Diagnosis of imported monkeypox, Israel, 2018. Emerg Infect Dis. 2019;25(5):980. (doi: 10.3201/eid2505.190076).

13. Simpson K, Heymann D, Brown CS, et al. Human monkeypox-After 40 years, an unintended consequence of smallpox eradication. Vaccine. 2020. (doi: 10.1016/j.vaccine.2020.04.062).

14. Ježek Z, Szczeniowski M, Paluku KM, et al. Human monkeypox: clinical features of 282 patients. J Infect Dis. 1987;156(2):293-8. (doi: 10.1093/infdis/156.2.293).

15. Likos AM, Sammons SA, Olson VA, et al. A tale of two clades: monkeypox viruses. J Gen Virol. 2005;86(10):2661-72. (doi: 10.1099/vir.0.81215-0).

16. Chen N, Li G, Liszewski MK, et al. Virulence differences between monkeypox virus isolates from West Africa and the Congo basin. Virology. 2005;340(1):46-63. (doi: 10.1016/j.virol.2005.05.030).

17. Sbrana E, Xiao SY, Newman PC, Tesh RB. Comparative pathology of North American and central African strains of monkeypox virus in a ground squirrel model of the disease. AmJ Trop. Med Hyg. 2007;76:155-64.

18. Petersen E, Kantele A, Koopmans $M$, et al. Human monkeypox: epidemiologic and clinical characteristics, diagnosis, and prevention. Infect Dis Clin. 2019;33(4):1027-43. (doi: 10.1016/j.idc.2019.03.001).

19. Tom JJ, Anebo NP. A Neuro-Fussy Based Model for Diagnosis of Monkeypox Diseases. 2018.

20. Yinka-Ogunleye A, Aruna O, Dalhat M, et al. Outbreak of human monkeypox in Nigeria in 2017-18: a clinical and epidemiological report. Lancet Infect Dis. 2019;19(8):872-9. (doi: 10.1016/S1473-3099(19)30294-4).

21. Faye $O$, Pratt $C B$, Faye $M$, et al. Genomic characterisation of human monkeypox virus in Nigeria. Lancet Infect Dis. 2018;18(3):246. (doi: 10.1016/S1473-3099(18)30043-4).

22. Centers for Disease Control and Prevention (CDC). Multistate outbreak of monkeypox--Illinois, Indiana, and Wisconsin, 2003. MMWR. Morbidity and mortality weekly report. 2003;52(23):537. 
23. Bartlett J. Monkeypox Review. Medscape. 2003. [cited 2021 Jan 2]. Available at: https://www.medscape.com/ viewarticle/458671_11

24. Damon IK. Status of human monkeypox: clinical disease, epidemiology and research. Vaccine. 2011;29:D54-D59. (doi: 10.1016/j.vaccine.2011.04.014).

25. Reynolds MG, Carroll DS, Karem KL. Factors affecting the likelihood of monkeypox's emergence and spread in the post-smallpox era. Curr Opin Virol. 2012;2(3):335-43. (doi: 10.1016/j.coviro.2012.02.004).

26. Fauci A, Robert H. Ebert Memorial Lecture-Emerging and Re-emerging Infectious Diseases: The Perpetual Challenge. Milbank Memorial Fund; 2015. [cited 2020 Oct 28]. Available at: https://www.milbank.org/wpcontent/uploads/2016/04/0601Fauci.pdf

27. Bed TTO, Adesina MA. Sakkiya practice in the 'north:'A gaze into the past, the present, and the future. J Altern Med Res. 2018;10(4):315-22.

28. Adesina MA, Kanmodi KK, Fagbule OF, Bello. Sakkiya and orthodox medicine in Nigeria: A compared narrative. J Altern Med Res. 2018;10(4):341-6.

29. Bello A, Adesina MA, Kanmodi KK. Northern Nigeria: An overview. In Kanmodi KK, J Merrick (Eds). The traditional Sakkiya practice: A public health issue in Northern Nigeria. New York, NY: Nova Science. 2019;3-16.

30. Sklenovská N, Van Ranst M. Emergence of monkeypox as the most important orthopoxvirus infection in humans. Front Public Health. 2018;6:241. (doi: 10.3389/fpubh.2018.00241).

31. CDC, Centers for Disease Control and Prevention. Monkeypox. 2015. [cited 2020 Oct 25]. Available at: https://www.cdc.gov/poxvirus/monkeypox/symptoms. html

32. World Health Organization (WHO). Monkeypox. 2019. [cited 2020 Oct 26]. Available at: https://www.who. int/news-room/fact-sheets/detail/monkeypox

33. Karem KL, Reynolds M, Braden Z, et al. Characterization of acute-phase humoral immunity to monkeypox: use of immunoglobulin $M$ enzyme-linked immunosorbent assay for detection of monkeypox infection during the 2003 North American outbreak. Clin Diagn Lab Immunol. 2005;12(7):867-72. (doi: 10.1128/CDLI.12.7.867872.2005).
34. Pal M, Mengstie F, Kandi V. Epidemiology, Diagnosis, and Control of Monkeypox Disease: A comprehensive Review. Am J Infect Dis. 2017;5(2):94-9. (doi: 10.12691/ajidm-5-2-4).

35. Russo AT, Berhanu A, Bigger CB, et al. Co-administration of tecovirimat and $A C A M 2000^{\mathrm{TM}}$ in non-human primates: Effect of tecovirimat treatment on ACAM2000 immunogenicity and efficacy versus lethal monkeypox virus challenge. Vaccine. 2020;38(3):644-54. (doi: 10.1016/j.vaccine.2019.10.049).

36. FDA. FDA approves first live, non-replicating vaccine to prevent smallpox and monkeypox; FDA News Release; September, 2019. [cited 2020 Oct 19]. Available at: https://www.fda.gov/news-events/pressannouncements/fda-approves-first-live-non-replicatingvaccine-prevent-smallpox-and-monkeypox

37. Petersen BW, Kabamba J, McCollum AM, et al. Vaccinating against monkeypox in the Democratic Republic of the Congo. Antiviral Res. 2019;162:171-7. (doi: 10.1016/j.antiviral.2018.11.004).

38. World Health Organization (WHO). Monkeypox Democratic Republic of the Congo. WHO Disease outbreak news, October. 2020. [cited 2020 Oct 28]. Available at: https://www.who.int/csr/don/01-october2020-monkeypox-drc/en/

39. Adesina MA, Olufadewa II, OgaH YI, Nwachukwu N. Incidence and mortality from a neglected tropical disease (Rabies) in 28 African countries. Folia Vet. 2020;64(2):46-51. (doi: 10.2478/fv-2020-0016).

40. Olufadewa II, Adesina MA, Ayorinde T. From Africa to the World: Reimagining Africa's research capacity and culture in the global knowledge economy. J Glob Health. 2020a;10(1). (doi: 10.7189/jogh.10.010321).

41. Olufadewa II, Adesina MA, Ayorinde T, Oladele RI. Reimagining One Health in Africa: a strategy to ending re-emerging infections and anti-microbial resistance. Int J Health Plann Manage. 2020b. (doi: 10.1002/hpm.3069). 